\title{
Mobile radio services for urban transport
}

\author{
C. Bantin \\ Alcatel, Canada
}

\begin{abstract}
Mobile IP networks, using wireless LAN (WLAN) technology, have recently been successfully deployed for communications based train control (CBTC) applications. Packet transmission networks are well suited to the data communications requirements of CBTC, and WLANs, using universal data packet (UDP) transmission, a simple send and forget protocol, are ideally suited for this task. Moreover, the 802.11 family of WLAN radio standards allows for several megabits per second of data transmission, whereas automatic control applications require at most 10 to $20 \mathrm{~kb} / \mathrm{s}$ data links. This leaves ample capacity for carrying additional data to and from transit vehicles, and opens up the possibility of providing additional data services. Transit operators are becoming increasingly interested in utilizing this extra capacity for CCTV, voice and emergency response services. WLAN radios can carry varying amounts of data for these additional services from one CCTV signal plus voice and data for basic 802.11 , to several high-quality video signals and voice circuits for $802.11 \mathrm{~g}$ systems.
\end{abstract}

\section{Introduction}

Within the past two years, two urban transport rail systems have gone into revenue service using radio-based communications to support automatic train control (ATC) operations with driverless trains. The first is the Las Vegas monorail [1] and the second is the Disney Resort Line in Hong Kong. These are the first such revenue systems in the world to utilize radio for their ATC operation. The radio networks are based on the IEEE 802.11 [2-4] WLAN standards for communication between the trains and the wayside.

CBTC systems have a modest requirement for data rate but stringent requirements for availability and delay. The ATC equipment will communicate with each train by polling it and expecting a response within a certain amount of 
time. Therefore, although it is not necessary to have continuous communications with a train, it is important that the radio links be available when they are needed, and not to delay any response. Furthermore, it is important to maintain this availability and quick response time while the trains are moving at speeds of over $100 \mathrm{~km} / \mathrm{h}$.

So far, the only type of network that can meet all of these requirements is a wireless local area network or WLAN. These networks are the mobile extension of the familiar LANs that are extensively used for Ethernet communications, such as the Internet. LANs utilize a particular access method that permits all users to share one high capacity circuit. WLANs offer this same access method over a wireless connection, suitably modified for the wireless environment. The capacity of such WLANs, however, is a lot higher than is required just by CBTC, or indeed that which would be required for automatic operation of other types of transit vehicles. There is considerable excess capacity over the radios that can be used to provide other services. Meanwhile, transit operators are showing an increasing interest in providing digital services to and from their vehicles. The most important of these is CCTV for real time security monitoring of train cars, emergency response voice circuits, and passenger information services.

\section{Radio network requirements for CBTC}

The radio-based CBTC networks currently in revenue service are primarily designed to meet the requirements of the ATC system. One important requirement is for a high availability of the communications links between the radios. This requirement dictates the locations of the wayside radio access point equipment and other parameters of the radio network design, such as the need for redundant coverage. Another important requirement is for the train radios to be able to be able hand-over communications to successive access points as it travels along the guideway at speeds that can reach over $100 \mathrm{~km} / \mathrm{h}$. Typical data communications between a train and wayside equipment consists of data packets of about 100 bytes in size transmitted several times per second in both directions. This amounts to a modest few kilobits per second in data throughput, but it must be achieved with a latency, or transmission delay, of less than a few tens of milliseconds for any one packet. On the other hand, the integrity of the train control messages is maintained by checks from within the ATC equipment itself, therefore there is no need for ensuring one hundred percent data integrity at the radio transmission level.

These requirements are best met using a WLAN solution. The open-standard for WLAN networks is the IEEE 802.11 [2-4]. Furthermore, WLAN radios using the 802.11 standard, operate in license-exempt bands at 2.4 or $5.8 \mathrm{GHz}$. All WLAN users of these bands employ a standard access protocol, which is a self-regulating contention access method and allows many users to share the band simultaneously. For a lot of cases a $802.11 \mathrm{~b}, \mathrm{~g}$ (WiFi) solution will work well. Nevertheless, the presence of additional non-WLAN interference cannot be mitigated by the access protocol. In these cases a frequency-hopping spread spectrum (FHSS) version of 802.11 is best. This provides the added protection of 
being able to hop away from any specific instances of interference, especially deliberate interference from an intruder. While $802.11 \mathrm{~b}$ and $11 \mathrm{~g}$ offer a transmission capacity of up several megabits per second, the frequency-hopping variant is limited to about one megabit per second.

Figure 1 illustrates how a radio-based network is laid out along a transit guideway, for example the Disney Resort Line at Penny's Bay in Hong Kong.

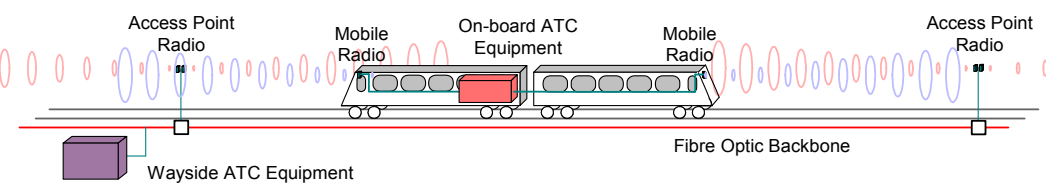

Figure 1: $\quad$ Radio CBTC deployed along a guideway.

Mobile radios on the vehicle, which are connected to the on-board ATC equipment, communicate through access point radios along the wayside, which in turn are connected to the wayside ATC equipment through a fibre optic Ethernet backbone. Continual communications is maintained along the guideway by properly locating the access points.

\section{Radio network data rates for other services}

Given the high availability of the CBTC WLAN radio system along the entire guideway, and the excess capacity over what is required for ATC, it is natural for system operators to look at adding other data services to the radio system. Today, just about any type of communications can be carried in digital form, however of the most interest to operators are CCTV, voice circuits and data for passenger information display systems. The data rate requirements for these services vary widely.

\subsection{CCTV}

Currently, video signals from an on-board CCTV camera are digitized and stored on-board because there has been no high capacity link available to monitor them in real time. Recordings are retrieved and reviewed for evidence after the fact. However, with the advent of high-speed digital radio links it is now possible for the camera signals from inside a vehicle to be viewed in real time along with those from station platforms.

The data communications requirements for CCTV transmissions are varied. First the signal from a camera is digitized. Then the raw data signal is compressed from several megabits per second to as low as a few hundred kilobits per second. The most recent standard for compressing the signals comes from the motion picture experts group and is known as MPEG4 [5]. The data rates depend on two main factors, the number of frames displayed per second and the 
resolution of each frame. The frame resolution is stated in terms of CIF (or common intermediate format), which was established in the early days of video conferencing. A resolution of 1 CIF corresponds to a pixel resolution of 352 by 288 in PAL or $352 \times 240$ in NTSC. Therefore it is roughly equivalent to $1 / 4$ of the basic PC screen resolution of 640 x 480 pixels. Today it is common to specify at least a 4 CIF resolution, which amounts to $704 \times 480$ pixels and the image is typically displayed in about $1 / 4$ of a high-resolution (1024 x 768 or more) monitor. At the same time it is not necessary to have a high frame repetition rate for CCTV surveillance purposes. Many current CCTV systems offer less than 1 frame per second, however a good compromise for catching all the action is 4 to 6 frames per second (fps).

While medium resolution and low frame repetition rates may be sufficient for most CCTV applications, there can also be a requirement for higher resolution and faster repetition rates. So-called "broadcast quality" video used for standard definition television requires at least $30 \mathrm{fps}$.

When it comes to transmitting this data, special coding is used so that only the changes from one frame to the next are sent. Scenes that contain no motion typically require very little new data because there is nothing changing. Conversely scenes that change rapidly require a much larger amount of new data. The trick is to buffer the signals so that the data transmission rates can be smoothed out to some acceptable average. For example a 4 CIL, 6 fps CCTV signal can be transmitted at between $500 \mathrm{~kb} / \mathrm{s}$ and $900 \mathrm{~kb} / \mathrm{s}$. Broadcast quality video requires at least $2 \mathrm{Mb} / \mathrm{s}$. A definite advantage of using a WLAN network is that it can readily and efficiently accept the varying data transmission rates presented by the video compression equipment.

\subsection{Voice}

Voice circuits for communication between drivers and other personnel on the wayside have been implemented for some time now using two-way push-to-talk radios or cell phone circuits. Now, with the unused data transmission capacity available on a vehicle equipped with a radio-based CBTC, this and many new voice functions can be accommodated on the network radios. One of the more commonly requested services is emergency response. At the push of a button any passenger can have a voice circuit for emergency use to a central operator or the driver. In fact this action can be coupled with the CCTV system so that the nearest camera can be trained onto the passenger as the voice circuit is activated. Data requirements for voice are quite modest relative to video pictures. Digitized voice signals can be transmitted at a few $10 \mathrm{~s}$ of kilobits per second. Furthermore, the emerging voice-over-IP (VoIP) technology can be used to carry the voice over the WLAN network without further modification, and the voice can be automatically addressed to a specific destination by using its IP address.

\subsection{Data}

The most common use for data services to and from vehicles is for passenger information display purposes. For this application data transmission would not 
be continuous but would only be required to update display status like the next station name and transfer information. An aggregate transmission rate in the order of $20 \mathrm{~kb} / \mathrm{s}$ would be more than sufficient.

\section{Capacity of WLAN radio networks}

The capacity of a complete CBTC data communications system (DCS) network is limited by the capacity of the individual 802.11 radio links. This, in turn, depends on the version of the standard that is implemented. Basic frequency hopping or direct sequence spread spectrum systems (802.11 FHSS or DSSS) transmit at 1 or $2 \mathrm{Mb} / \mathrm{s}$, while $\mathrm{WiFi}$ systems operate from 11 and $22 \mathrm{Mb} / \mathrm{s}$ (for $802.11 \mathrm{~b}$ ) up to $54 \mathrm{Mb} / \mathrm{s}$ (for $802.11 \mathrm{~g}$ ). A given radio link, however, cannot achieve these rates as data throughput. This is because of two factors; the overhead contained within the IP packets and the network management, and the contention access methods of the 802.11 standard. These systems were designed to allow many users to share access to the radio band through contention. When there are many users there is an increased risk of collisions and packets need to be resent. The theoretical maximum achieved throughput for a large number if independent users is statistically about $37 \%$ of the actual transmission rate. In practice this would be closer to $30 \%$. Under these conditions over $2 / 3$ of the packets transmitted are actually retries following earlier collisions. Therefore, ten simultaneous users of a $2 \mathrm{Mb} / \mathrm{s} 802.11 \mathrm{FHSS}$ system (i.e. one radio link) may achieve an aggregate of $0.6 \mathrm{Mb} / \mathrm{s}$ throughput, or $60.0 \mathrm{~kb} / \mathrm{s}$ per user. For a $54 \mathrm{Mb} / \mathrm{s} 11 \mathrm{~g}$ system this would be $1.6 \mathrm{Mb} / \mathrm{s}$ per user. In a system designed for CBTC operation along a guideway the number of users is much smaller and often is only one per AP radio. Under these conditions the user gets to use about $70 \%$ of the transmission rate, or about $1.4 \mathrm{Mb} / \mathrm{s}$ for the FHSS system. A CBTC system which needs perhaps $20 \mathrm{~kb} / \mathrm{s}$ per user (vehicle radio) can easily operate in these environments, and the radios have anywhere from $650 \mathrm{~kb} / \mathrm{s}$ to many $\mathrm{Mb} / \mathrm{s}$ excess capacity. Figure 2 illustrates, on a logarithmic scale, how much additional voice, data and CCTV services can be carried by the various implementations of a WLAN network.

A basic $1 \mathrm{Mb} / \mathrm{s}$ FHSS system, which has the highest degree of interference protection, can readily carry passenger information data, one voice circuit and one CCTV signal through each radio link. A higher capacity $2 \mathrm{Mb} / \mathrm{s}$ FHSS system can handle this traffic plus a second CCTV signal per radio. It should be noted that a vehicle, such as a subway train, has separate radios at each end of the train. This is primarily for redundancy in order to provide a high availability for ATC traffic. However, each radio can carry separate CCTV signals. This will allow a $2 \mathrm{Mb} / \mathrm{s}$ FHSS system to carry at least four, and perhaps up to six, separate CCTV signals. If priority is given to any one particular camera then the high availability can be maintained for emergency purposes. Redundancy can be included by connecting the radios at each end of the vehicle to a common LAN bus. 
The higher throughput rates available from the $11 \mathrm{~b}$ or $11 \mathrm{~g} \mathrm{WiFi}$ radios do not come free. In order to achieve the same packet loss rate as a FHSS system, a WiFi mobile radio would need a stronger signal and this translates to more APs, spaced closer together, along the guideway.

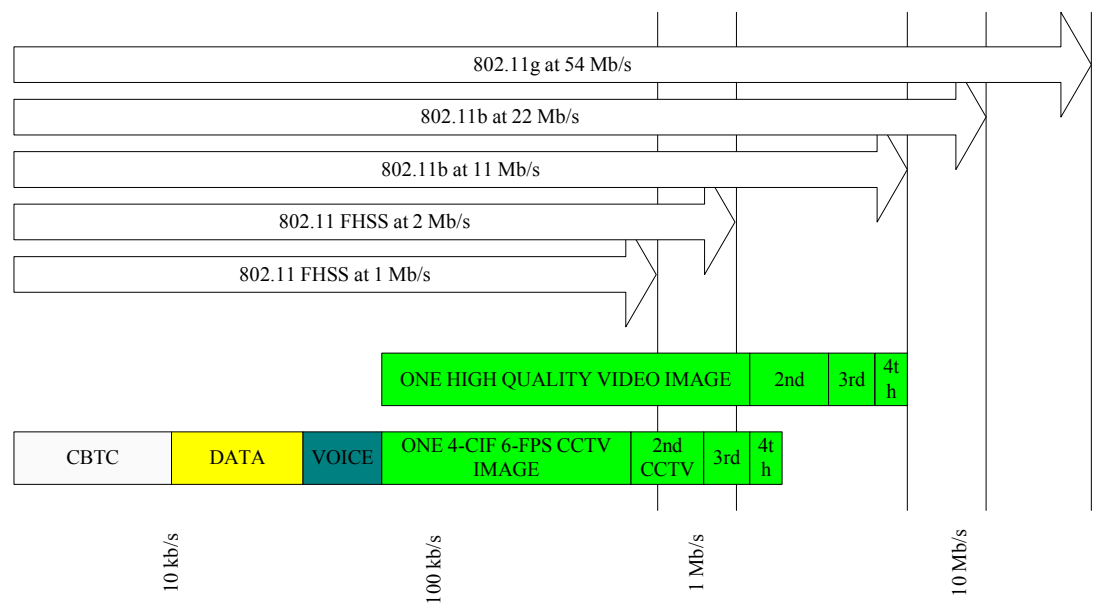

Figure 2: $\quad$ Data carrying capabilities of 802.11 WLAN Networks.

\section{Conclusions}

WLAN networks based on the 802.11 standards are ideal for carrying the communications traffic for ATC purposes. In addition, the excess capacity of these radios allows for a significant amount of additional data services to be carried. These services include CCTV, voice circuits and data for passenger information services. A basic 802.11 frequency hopping radio can carry two high-quality CCTV images, voice circuits and data. Other versions of the standard, $802.11 \mathrm{~b}$ or g, popularly known as WiFi, can carry many more simultaneous CCTV images and/or several broadcast quality video images.

\section{References}

[1] Bantin C., Siu J., Radio CBTC for the Las Vegas Monorail, Urban Transport XI, Brebbia C.A., Wadhwa L.C. editors, WIT Press, 2005, pp95 103

[2] ANSI/IEEE Std 802.11, 1999 Edition, Information technology Telecommunications and information exchange between systems - Local and metropolitan area networks - Specific requirements, Part 11: Wireless LAN Medium Access Control (MAC) and Physical Layer (PHY) Specifications

[3] ibid: Higher-Speed Physical Layer Extension in the $2.4 \mathrm{GHz}$ Band

[4] ibid: Higher-Speed Physical Layer Extension in the $5 \mathrm{GHz}$ Band

[5] http://www.chiariglione.org/mpeg/standards/mpeg-4/mpeg-4.htm 